\title{
A Remark on \\ Yukawa Plus Boson Selfinteraction in Two Space Time Dimensions*
}

\author{
ROBERT SCHRADER \\ Lyman Laboratory of Physics, Harvard University, Cambridge, Massachusetts
}

Received October 20, 1970

\begin{abstract}
In this note we show how the results of Glimm and Jaffe $[5,6]$ on the Yukawa quantum field theory in two space time dimensions may be extended to the case where a boson selfinteraction term is added. Local fields are constructed which do not depend on any cut-off and which have the right (anti)-commutation properties for spacelike separated support of the test functions.
\end{abstract}

\section{Introduction}

Throughout this paper we shall employ the notations and definitions used in [7] to discuss the Yukawa interaction in two space dimensions. Let

$$
H^{Y}(g, x)=H_{0}+H_{I}(g, \varkappa)+c(g, x)
$$

be the cutoff Hamilton operator describing the Yukawa interaction between a boson field $\varphi$ and a fermion field $\psi \cdot g$ is a nonnegative space cutoff function and $x$ a momentum cutoff parameter. $c(g, x)$ is a renormalization counterterm. Set

$$
: P(\varphi)(g):=\sum_{r=0}^{2 n} a_{r} \int: \varphi(x)^{2 n}: g(x) d x
$$

where all $a_{r}$ are real and $a_{2 n}>0$. Define

$$
H(g, \varkappa)=H^{Y}(g, \varkappa)+: P(\varphi)(g):
$$

Note that :P( $\varphi(g)$ : has only a space cutoff and no momentum cutoff. We shall be considering the family $H(g, x)$ as $x \rightarrow \infty$. It will be proved that the resolvents of $H(\mathrm{~g}, \varkappa)$ converge to the resolvent of a selfadjoint Hamilton operator $H(g)$, which locally gives rise to a finite propagation speed.

Further let

$$
\begin{gathered}
\psi(f)=\int e^{i t \boldsymbol{H}(g)} \psi(x) e^{-i t \boldsymbol{H}(g)} f(x, t) d x d t, \\
\varphi(f)=\int e^{i t \boldsymbol{H}(g)} \varphi(x) e^{-i t \boldsymbol{H}(g)} f(x, t) d x d t\left(f \in C_{0}^{\infty}\left(\mathbb{R}^{2}\right)\right)
\end{gathered}
$$

* Supported in part by the Air Force Office of Scientific Research, Contract F 44620-70-C-0030. 
be the smeared out field operators. Then $\psi(f)$ is a bounded operator and $\varphi(f)$ ( $f$ real) a selfadjoint operator. $\psi(f)$ and $\varphi(f)$ will be independent of $g$, if $g=1$ on a sufficiently large set. Finally these fields are shown to have the right (anti-)commutation properties for space like separation of the support of the test functions.

\section{First and Second Order Estimates}

The results of $[2,3,9,10]$ easily show the following

Theorem 2.1. For each $x<\infty, H(g, x)$ is selfadjoint and e.s.a. (essentially selfadjoint) on $C^{\infty}\left(H_{0}\right)$. In fact any core for $\left(H_{0}\right)^{n}$ is a core for $H(g, \varkappa)$. Also

$$
N_{\tau} \leqq \operatorname{const}(H(g, \varkappa)+\text { const }) \quad \tau<1
$$

with constants independent of $x$.

Hence we may choose $c(g, x)$ in such a way that

$$
\text { inf spectrum } H(g, x)=0 \text {. }
$$

Also using (2.1) we may establish pull-through fourmulas similar to those given in [5] and [10]. This will give

Theorem 2.2. For $\tau<\frac{1}{2}$ the following quadratic $N_{\tau}$ estimate holds

$$
N_{\tau}^{2} \leqq \text { const }(H(g, x)+\text { const })^{2}
$$

with constants independent of $x$.

To prove inequality (2.3), the estimate

$$
N_{\tau}^{2} \leqq \text { const }\left(H^{Y}(g, \varkappa)+\text { const }\right)^{2} \quad\left(\tau<\frac{1}{2}\right)
$$

with constants independent of $\chi$, is necessary. The estimate (2.4) however, was established by Dimock [1].

The proof of Theorem 2.2 is quite long, but since no new techniques are needed, no details will be presented here.

\section{The Local Hamiltonian}

The estimates (2.1) and (2.3) permit the proof of the following

Theorem 3.1. As $x \rightarrow \infty$ the resolvents $R_{x}(\zeta)$ of $H(g, x)$ converge uniformly to the resolvent $R(\zeta)$ of a selfadjoint nonnegative operator $H(g)$. Zero is an isolated eigenvalue of $H(g)$.

12 Commun. math. Phys., Vol. 21 
Indeed we have

where

$$
R_{\varkappa}(\zeta)-R_{\chi^{\prime}}(\zeta)=-R_{\varkappa}(\zeta) \delta H R_{\chi^{\prime}}(\zeta)
$$

$$
\begin{aligned}
\delta H & =H(g, \varkappa)-H\left(g, \varkappa^{\prime}\right) \\
& =H^{Y}(g, \varkappa)-H^{Y}\left(g, \varkappa^{\prime}\right)
\end{aligned}
$$

and hence, using the pull through formulas, essentially the same arguments may be used as in the discussion of $H^{Y}(g, x)$ [5-7]. Also

$$
\begin{aligned}
N_{2 \tau} & \leqq \text { const }(H(g)+\text { const }) \quad \tau<\frac{1}{2}, \\
\left(N_{\tau}\right)^{2} & \leqq \text { const }(H(g)+\text { const })^{2} .
\end{aligned}
$$

Let $\mathscr{A}_{f}(B)($ resp. $\mathscr{A}(B))$ be the time zero field algebra (resp. observable algebra) of [7], where $B$ is a bounded open set in $\mathbb{R}$. Also let $B_{t}$ be the set of points in $R$ with distance less than $|t|$ from $B$.

Theorem 3.2. For $g=1$ on $B_{t}$, the map $A \rightarrow A(t)$ with

$$
A(t)=e^{i t H(g)} A e^{-i t H(g)}
$$

defines a g-independent automorphism

$$
\begin{aligned}
& \sigma_{t}: \mathscr{A}_{f}(B) \rightarrow \mathscr{A}_{f}\left(B_{t}\right), \\
& \sigma_{t}: \mathscr{A}(B) \rightarrow \mathscr{A}(B),
\end{aligned}
$$

i.e. $H(g)$ satisfies the finite propagation speed property.

The proof is essentially as in [6], since $H_{0}+: P(\varphi)(g)$ : satisfies the finite propagation speed property [9].

\section{The Field Operators}

The operators $\psi(f)$ in (1.4a) make no real difficulties since they are fermion operators. They are bounded and independent of $g$, if $g=1$ on a sufficiently large set due to Theorem 3.2. Now let $f \in C_{0}^{\infty}\left(\mathbb{R}^{2}\right)$ be real and put

$$
\varphi_{g}\left(f_{t}\right)=\int e^{i t \boldsymbol{H}(g)} \varphi(x) e^{-i t \boldsymbol{H}(g)} f(x, t) d x .
$$

Using the estimates (3.3) and (3.4) and arguments of [4,5], we have the

Theorem 4.1. $\varphi_{g}\left(f_{t}\right)$ is a selfadjoint operator, e.s.a. on $D\left((H(g)+1)^{\frac{1}{2}}\right)$ and for $\Theta \in D\left((H(g)+1)^{\frac{1}{2}}\right), \varphi_{g}\left(f_{t}\right) \Theta$ is strongly continuous in $t$, with the estimates

$$
\begin{aligned}
\left\|\varphi_{g}\left(f_{t}\right) \Theta\right\| & \leqq \text { const }\|f(\cdot, t)\|_{2}\left\|(H(g)+1)^{\frac{1}{2}} \Theta\right\|, \\
\left\|\varphi_{g}\left(f_{t}\right)^{2} \Theta\right\| & \leqq \text { const }\|f(\cdot, t)\|_{2}^{2}\|(H(g)+1) \Theta\|
\end{aligned}
$$


Due to this theorem, the integral

$$
\varphi_{g}(f) \Theta=\int \varphi_{g}\left(f_{t}\right) \Theta d t
$$

exists and may be used to define $\varphi_{g}(f)$ as a closed symmetric operator

$$
\varphi_{g}(f)=\left(\left.\varphi_{g}(f)\right|_{D(H(g)+1)^{\frac{1}{2}}}\right)^{-}
$$

and we have the

Theorem 4.2. $\varphi_{g}(f)$ is selfadjoint and hence e.s.a. on any core of $(H(g)+1)^{\frac{1}{2}}$ since

$$
\left\|\varphi_{g}(f) \Theta\right\| \leqq \text { const } \int\|f(\cdot, t)\|_{2} d t\left\|(H(g)+1)^{\frac{1}{2}} \Theta\right\| .
$$

For $g=1$ on a sufficiently large set depending on $\operatorname{supp} f, \varphi_{g}(f)$ is actually independent of $g$.

The first part of the theorem may be proved as in [4]. For the second part, which is also a new result in the case of ordinary Yukawa coupling, we need two well known lemmata, which we state for convenience (see e.g. [8], pp. 429 and 432).

Lemma 4.3. Let $T_{n}$ and $T$ be selfadjoint operators in a Hilbert-space and let $D$ be a core of $T$ such that $T_{n} u \rightarrow T u$ as $n \rightarrow \infty$ for each $u \in D$. Then the resolvents of $T_{n}$ converge strongly to the resolvent of $T$.

Lemma 4.4. Let the hypotheses be as in Lemma 4.3 and let $E_{n}(\lambda)$ resp. $E(\lambda)$ be the spectral family of $T_{n}$ resp. T. Then

$$
\begin{aligned}
& s-\lim E_{n}\left(\lambda^{\prime}\right)=E(\lambda) \\
& \lambda^{\prime} \rightarrow \lambda \\
& n \rightarrow \infty
\end{aligned}
$$

if $E(\lambda)=E(\lambda-0)$.

We want to apply these lemmata in the following way. Define the selfadjoint operators

$$
\varphi\left(f_{t}\right)=\int \varphi(x) f(x, t) d x
$$

such that $\varphi\left(f_{t}\right)$ are all e.s.a. on $D\left((H(g)+1)^{\frac{1}{2}}\right)$ and

$$
\varphi_{g}\left(f_{t}\right)=e^{i t \boldsymbol{H}(g)} \varphi\left(f_{t}\right) e^{-i t \boldsymbol{H}(g)}
$$

Let $E\left(\lambda, f_{t}\right)$ be the spectral family of $\varphi\left(f_{t}\right)$. Then due to the local properties of $H(g)$

$$
E^{t}\left(\lambda, f_{t}\right)=e^{i t \boldsymbol{H}(g)} E\left(\lambda, f_{t}\right) e^{-i t \boldsymbol{H}(g)}
$$

does not depend on $g$, if $g=1$ on a sufficiently large set $B=B(f)$. Also using the canonical commutation relations in Weyl form of the time zero $12^{*}$ 
boson field and its canonically conjugate field, it is easy to see that

$$
E\left(\lambda, f_{t}\right)=E\left(\lambda-0, f_{t}\right)
$$

if $\varphi\left(f_{t}\right) \neq 0$ or $\lambda \neq 0$, i.e. $\varphi\left(f_{t}\right)$ is either zero or has no discrete eigenvalues. Therefore the above lemmata give

$$
\begin{aligned}
& s-\lim E\left(\lambda^{\prime}, f_{t^{\prime}}\right)=E\left(\lambda, f_{t}\right) \\
& t^{\prime} \rightarrow t \\
& \lambda^{\prime} \rightarrow \lambda
\end{aligned}
$$

if $\varphi\left(f_{t}\right) \neq 0$ or $\lambda \neq 0$. In particular

$$
C_{n}(f)=\int_{|\lambda| \leqq n} \lambda d E^{t}\left(\lambda, f_{t}\right) d t
$$

is a well defined bounded selfadjoint operator independent of $g$. We will show that the resolvents of $C_{n}(f)$ converge strongly to the resolvent of $\varphi_{g}(f)$. This will show that $\varphi_{g}(f)$ is actually independent of $g$. To prove the strong convergence of the resolvents, by the first part of the theorem and Lemma 4.3, it is sufficient to prove

$$
s-\lim _{n \rightarrow \infty} C_{n}(f) u=\varphi_{g}(f) u
$$

for $u \in D((H(g)+1))$. We have

$$
\begin{gathered}
\left\|\left(\varphi_{g}(f)-C_{n}(f) u\right)\right\|=\left\|\int e^{i t H(g)}\left(\varphi\left(f_{t}\right)-\int_{|\lambda| \leqq n} \lambda d E\left(\lambda, f_{t}\right)\right) e^{-i t H(g)} d t u\right\| \\
\leqq \int\left\|_{|\lambda| \geqq n} \lambda d E\left(\lambda, f_{t}\right)\left(\varphi\left(f_{t}\right)^{2}+1\right)^{-1}\left(\varphi\left(f_{t}\right)^{2}+1\right)(H(g)+1)^{-1}(H(g)+1) e^{-i t H(g)} u\right\| d t \\
\leqq \sup _{t}\left\|\int_{|\lambda| \geqq n} \frac{\lambda}{\lambda^{2}+1} d E\left(\lambda, f_{t}\right)\right\| \int_{|t| \leqq T}\left(\varphi\left(f_{t}\right)^{2}+1\right)(H(g)+1)^{-1}\|d t\|(H(g)+1) u \|
\end{gathered}
$$

where $T$ is chosen so large that $f(\cdot, t)=0$ for $|t| \geqq T$. Now

and

$$
\sup _{t}\left\|\int_{|\lambda| \geqq n} \frac{\lambda}{\lambda^{2}+1} d E\left(\lambda, f_{t}\right)\right\| \leqq \frac{1}{n}
$$

by (4.3) so

$$
\int_{|t| \leqq T}\left\|\left(\varphi\left(f_{t}\right)^{2}+1\right)(H(g)+1)^{-1}\right\| d t<\infty
$$

$$
\left\|\left(\varphi_{g}(f)-C_{n}(f)\right) u\right\| \leqq \frac{\text { const }}{n}\|(H(g)+1) u\|
$$

Letting $n \rightarrow \infty$ proves Theorem 4.2. 
Remark 4.5. Inspection of the proof shows all that is needed is the validity of the two conditions

(a) first and second order $N_{\tau}$ estimates,

(b) finite propagation speed property of $H(\mathrm{~g})$.

Therefore Theorem 4.2 will hold any time these conditions are satisfied.

We now write $\varphi(f)$ instead of $\varphi_{g}(f)$.

Theorem 4.6. Let $f_{i}(i=1,2)$ be in $C_{0}^{\infty}\left(\mathbb{R}^{2}\right)$ such that $f_{1}$ and $f_{2}$ are real and have space-like separated support. Then $\varphi\left(f_{1}\right)$ commutes with $\varphi\left(f_{2}\right)$, and $\psi\left(f_{2}\right)$. Also $\psi\left(f_{1}\right)$ anticommutes with $\psi\left(f_{2}\right)$.

The proof may be taken over from [4]. It also follows easily from the proof of Theorem 4.2, indeed we have e.g.

$$
\left[C_{n}\left(f_{1}\right), C_{m}\left(f_{2}\right)\right]=0
$$

for all $n, m$ by construction and hence

$$
e^{i \tau_{1} C_{n}\left(f_{1}\right)} e^{i \tau_{2} C_{m}\left(f_{2}\right)}=e^{i \tau_{2} C_{m}\left(f_{2}\right)} e^{i \tau_{1} C_{m}\left(f_{1}\right)}
$$

for all $n, m ; \tau_{1}, \tau_{2} \in \mathbb{R}$.

Now due to the convergence of the resolvents

$$
\begin{aligned}
& s-\lim _{n \rightarrow \infty} e^{i \tau_{1} C_{n}\left(f_{1}\right)}=e^{i \tau_{1} \varphi\left(f_{1}\right)} \\
& S-\lim _{m \rightarrow \infty} e^{i \tau_{2} C_{m}\left(f_{2}\right)}=e^{i \tau_{2} \varphi\left(f_{2}\right)}
\end{aligned}
$$

uniformly for $\tau_{1}, \tau_{2}$ in bounded sets of $\mathbb{R}$ (see e.g. [8] p. 502). Hence taking the strong limit of (4.13) proves

$$
e^{i \tau_{1} \varphi\left(f_{1}\right)} e^{i \tau_{2} \varphi\left(f_{2}\right)}=e^{i \tau_{2} \varphi\left(f_{2}\right)} e^{i \tau_{1} \varphi\left(f_{1}\right)}
$$

for all $\tau_{1}, \tau_{2}$, i.e. the spectral families of $\varphi\left(f_{1}\right)$ and $\varphi\left(f_{2}\right)$ commute. The other cases may be discussed in a similar way.

Acknowledgements. It is a pleasure to thank J. Dimock and Prof. A. Jaffe for helpful remarks.

\section{References}

1. Dimock, J.: Field equations for the $Y_{2}$ theory. Harvard University thesis. To appear.

2. Glimm, J.: The Yukawa coupling of quantum fields in two dimensions. Commun. math. Phys. 6, 61-76 (1967).

3. - Boson fields with nonlinear selfinteraction in two dimensions. Commun. math. Phys. 8, 18-28 (1967).

4. - Jaffe, A.: A $\lambda \Phi^{4}$ quantum field theory without cutoffs II. Ann. Math. 91, 362-401 (1970). 
5. - - Selfadjointness of the $Y_{2}$ Hamiltonian. Ann. Phys. 60, 321-383 (1970).

6. - - The Yukawa ${ }_{2}$ quantum field theory without cutoffs. To appear.

7. _ - "Field Theory Models". Lecture notes delivered at the Summer School in Les Houches 1970. To appear.

8. Kato, T.: Perturbation theory of linear operators. Berlin-Heidelberg-New York: Springer 1966.

9. Rosen, L.: A $\lambda\left(\Phi^{2 n}\right)_{2}$ field theory without cutoffs. Commun. math. Phys. 16, 157-183 (1970).

10. - The $\left(\Phi^{2 n}\right)_{2}$ quantum field theory. Higher order estimates. Preprint.

\author{
Robert Schrader \\ Lyman Laboratory of Physics \\ Harvard University \\ Cambridge, Mass. 02138
}

